\title{
Preparation of small silicon carbide quantum dots by wet chemical etching
}

\author{
David Beke ${ }^{\text {a) }}$ \\ Institute for Solid State Physics and Optics, Wigner Research Centre for Physics, Hungarian Academy of Sciences, \\ H-1525 Budapest, Hungary; and Faculty of Chemical Technology and Biotechnology, Budapest University of \\ Technology and Economics, H-1111 Budapest, Hungary \\ Zsolt Szekrényes and István Balogh \\ Institute for Solid State Physics and Optics, Wigner Research Centre for Physics, Hungarian Academy of Sciences, \\ H-1525 Budapest, Hungary
}

Zsolt Czigány

Institute for Technical Physics and Materials Science, Research Centre of Natural Sciences, Hungarian Academy of Sciences, H-1525 Budapest, Hungary

Katalin Kamarás

Institute for Solid State Physics and Optics, Wigner Research Centre for Physics, Hungarian Academy of Sciences, H-1525 Budapest, Hungary

Adam Gali

Institute for Solid State Physics and Optics, Wigner Research Centre for Physics, Hungarian Academy of Sciences, H-1525 Budapest, Hungary; and Department of Atomic Physics, Budapest University of Technology and Economics, H-1111 Budapest, Hungary

(Received 30 March 2012; accepted 14 June 2012)

Fabrication of nanosized silicon carbide ( $\mathrm{SiC}$ ) crystals is a crucial step in many biomedical applications. Here we report an effective fabrication method of $\mathrm{SiC}$ nanocrystals based on simple electroless wet chemical etching of crystalline cubic SiC. Comparing an open reaction system with a closed reaction chamber, we found that the latter produces smaller nanoparticles (less than $8 \mathrm{~nm}$ diameter) with higher yield. Our samples show strong violet-blue emission in the $410-450 \mathrm{~nm}$ region depending on the solvents used and the size. Infrared measurements unraveled that the surface of the fabricated nanoparticles is rich in oxidized carbon. This may open an opportunity to use standard chemistry methods for further biological functionalization of such nanoparticles.

\section{INTRODUCTION}

The search for ideal in vivo luminescent biomarkers is a great challenge because there are stringent criteria to be fulfilled: the biomarkers should (i) be nontoxic and bioinert, (ii) be photostable, i.e., be nonbleaching, (iii) show no intermittency in luminescence, i.e., there should be no blinking, (iv) be small where the critical diameter is close to $6 \mathrm{~nm}$, (v) be producible in large amounts for biological studies. Organic dye molecules are well characterized and can be synthesized in large selection of excitation and emission wavelength range. Dye molecules fulfill criteria (iv)-(v) but they often bleach and are toxic because the visible emission comes from double bonds or aromatic bonds. ${ }^{1}$ Recently, small luminescent nanocrystals have been synthesized that are photostable. The most successful candidate out of above is

\footnotetext{
a) Address all correspondence to this author.

e-mail: beke.david@wigner.mta.hu

DOI: 10.1557/jmr.2012.223
}

cadmium selenide (CdSe) where the size of the particles could be well controlled. Depending on the size, CdSe nanocrystals emit light of various wavelengths due to quantum confinement of the host electron states, so they are called CdSe quantum dots (QDs). ${ }^{2} \mathrm{CdSe}$ and other compound semiconductor QDs contain heavy metals that are toxic. ${ }^{3}$ While the diameter of these QDs is in the range of $1-5 \mathrm{~nm}$, a protecting shell is needed to avoid toxicity and aggregation and to make these QDs highly stable in aqueous solution. The hydrodynamic size of core/shell QDs is typically larger than $20 \mathrm{~nm}$. In addition, they often blink upon excitation. ${ }^{2,3}$ These QDs fulfill criteria (ii) and (v) but fail the other ones, particularly, criterion (i) which can be the most important one for in vivo studies.

In this article, we investigate silicon carbide QDs that are among the best candidates to fulfill criteria (i)-(v). Bulk silicon carbide ( $\mathrm{SiC}$ ) is a chemically inert wide bandgap indirect semiconductor with excellent hardness and heat resistance. The porous form of $\mathrm{SiC}$ has already been successfully applied in bone tissues and dentistry, thus $\mathrm{SiC}$ is considered to be a bioinert material. ${ }^{4} \mathrm{SiC}$-based 
microelectromechanical systems (MEMS) were also studied, ${ }^{5}$ and successfully applied in living systems. ${ }^{6}$ On the nanoscale, $\mathrm{SiC}$ shows a quantum confinement effect ${ }^{7-10}$ and luminescence properties within the $400-460 \mathrm{~nm}$ emission range. ${ }^{1-13} \mathrm{SiC}$ nanocrystals of the size of 1-6 nm have been studied in living organisms and show effective luminescence without perturbing the living cells. ${ }^{14}$ They can be produced in sizes smaller than $10 \mathrm{~nm}$ and no protective shells are needed for making SiC QDs biocompatible or for stabilizing them in aqueous systems. ${ }^{15}$

There are many ways to produce $\mathrm{SiC}$ QDs. SiC QDs may be fabricated by embedding $\mathrm{SiC}$ particles in $\mathrm{Si}$ or $\mathrm{SiO}_{2}$ matrix ${ }^{16}$ or by use of sol-gel precursors. ${ }^{17}$ Nevertheless, electrochemical etching of bulk $\mathrm{SiC}$ is the most widely used method where porous layers form during etching and these layers are broken down in an ultrasonic bath or by milling, and finally SiC QDs are produced with sizes smaller than $10 \mathrm{~nm} .{ }^{18}$ Cubic SiC may be effectively treated by electroless wet chemical etching in a mixture of hydrofluoric acid $(\mathrm{HF})$ and nitric acid $\left(\mathrm{HNO}_{3}\right)$ at elevated temperature. ${ }^{19}$ Recently, we were able to produce luminescent $\mathrm{SiC}$ QDs by this inexpensive method where the maximum size of $\mathrm{SiC}$ QDs was about $16 \mathrm{~nm}^{20}$ that did not fulfill criterion (iv). In this article, we report progress in controlling the size of $\mathrm{SiC}$ QDs by using a more sophisticated method in the production process and optimizing the parameters of the etching procedure where the produced $\mathrm{SiC}$ nanoparticles have come down to the desired size of QDs. We also analyze the surfaces of our prepared $\mathrm{SiC}$ QDs that have serious implications and potentialities for biological functionalization.

\section{EXPERIMENT}

Silicon (Across, 365 mesh) and graphite powder were successfully ground and pressed into a pellet, then heated in an induction chamber to produce $\mathrm{SiC}$ microcrystalline (MCs) powder. For cleaning the $\mathrm{SiC}$ powder from $\mathrm{Si}$ and $\mathrm{C}$ residue, the sample was etched in $\mathrm{HF}(50 \%)$ and $\mathrm{HNO}_{3}$ $(68 \%)$ and water mixture with volume ratio of 2:1:5 at ambient temperature, followed by washing with deionized water. Next, the dried sample was annealed at $900{ }^{\circ} \mathrm{C}$ for $6 \mathrm{~h}$ and the etching and drying step was repeated.

For obtaining the highest yield, about $2.0 \mathrm{~g}$ clean $\mathrm{SiC}$ powder was placed into a $23 \mathrm{~mL}$ Teflon-lined acid digestion bomb (Parr Instrument Company, Moline, IL) and annealed at $120{ }^{\circ} \mathrm{C}$ for $2 \mathrm{~h}$. Using this closed reaction chamber, the concentration of the acid mixture does not change because of the different vapor pressures of HF and $\mathrm{HNO}_{3}$, while temperature is controllable in a wider range. The open system was a perfluoroalkoxy (PFA) flask with 5-mm aperture that was connected to a reflux column and heated in a $120{ }^{\circ} \mathrm{C}$ oil bath. $2.0 \mathrm{~g} \mathrm{SiC}$ was etched for $2 \mathrm{~h}$, then in both cases the etching acid was decanted after centrifugation and $\mathrm{SiC}$ was washed with distilled water and dried. To produce SiC QDs, the acid-treated samples were sonicated in a chosen solvent for $1 \mathrm{~h}$ and centrifuged at $4000 \mathrm{rpm}$ for $1.5 \mathrm{~h}$. The supernatant fluid contains $\mathrm{SiC}$ QDs.

For characterizing the etching process, we weighed the sample before and after acid treatment and determined the yield of SiC QDs after the process as well. The size distribution was deduced from several high-resolution transmission electron microscopy (HRTEM) images where the particles were dried on an amorphous carbon (C)-coated TEM grid using a JEOL JEM-3010 HRTEM (Tokyo, Japan; $300 \mathrm{kV} ; 1.7 \AA$ resolution). Photoluminescence (PL) spectra were recorded in aqueous and ethanol solution using a Horiba Jobin-Yvon Nanolog spectrometer (Horiba Scientific, Edison, NJ). For characterizing the surfaces structure of the SiC QDs, Fourier transform infrared spectroscopy (FTIR) was used [Bruker Tensor 37 spectrometer, Bruker Optik GmbH, Ettlingen, Germany, equipped with an overhead attenuated total reflectance (ATR) unit for liquids].

\section{RESULTS AND DISCUSSION}

We successfully produced ultrasmall colloid SiC nanocrystals. After $2 \mathrm{~h}$ of etching the measured weight loss was $50 \mathrm{mg} / \mathrm{g}$ in both cases and the yield of SiC QDs was about $2 \mathrm{mg}$ for the open system and 3.0-3.5 mg when using a digestion bomb for the etching process. Thus, the closed reaction chamber can improve the yield with respect to the open system, which is an important issue. As a consequence, the intensity of photoluminescence also increases (see photoluminescence study below). The HRTEM image revealed that the SiC QDs were nearly spherical with size distribution of 1-8 $\mathrm{nm}$ and the average diameter was about $3 \mathrm{~nm}$ (see Fig. 1). Neither SiC QDs larger than $8 \mathrm{~nm}$ nor aggregated particles were found in any of the samples. This shows that the closed reaction chamber method leads to smaller $\mathrm{SiC}$ nanoparticles. This is a crucial improvement with respect to our previous study ${ }^{20}$ where

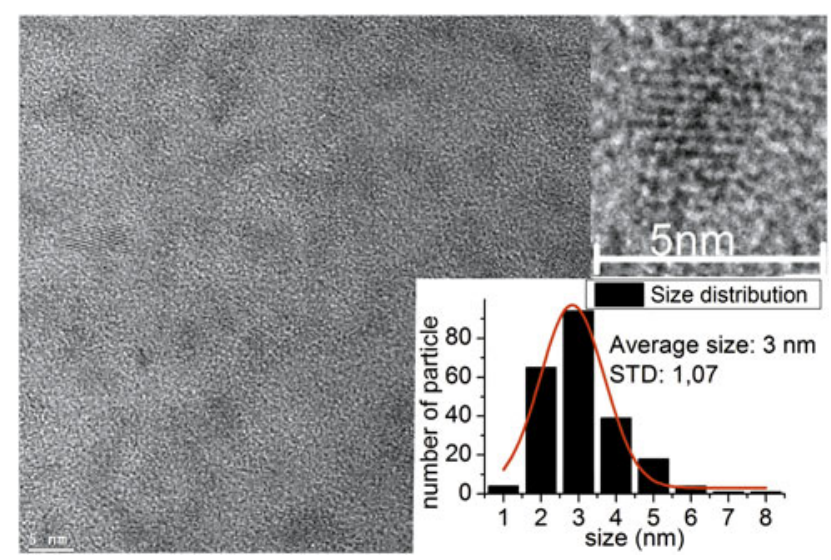

FIG. 1. HRTEM image (left and upper right above) and size distribution of $\mathrm{SiC}$ QDs (right below). The red line is a fitted Gaussian curve. 
we applied an open vessel system, resulting in a larger average diameter of about $6 \mathrm{~nm}$ and nanocrystals larger than $10 \mathrm{~nm}$ in the colloid suspension. By applying a closed reaction chamber and elevated temperature for annealing, the average size of $\mathrm{SiC}$ QDs could be reduced to $3 \mathrm{~nm}$ which fulfilled criterion (iv), and we found only few nanocrystals larger than $6 \mathrm{~nm}$ in diameter; their size $(7-8 \mathrm{~nm})$ is still close to the desired value. Our methodology is a promising route to ultimately fulfill criterion (iv).

Briefly, the two major advantages of the closed reaction system compared with the open vessel system are evident: larger output and narrower size distribution with smaller average diameter of the SiC QDs. In a closed system, neither the acid vapors nor gas phase products can escape during the reaction while high pressure is formed inside the reactor. We use an acid digestion bomb as a reactor and these bombs are validated up to $13 \mathrm{MPa}$. For getting more insight into the differences between the open and closedsystem reaction mechanism, we have to investigate the $\mathrm{SiC}$ etching mechanism. Wet etching of $\mathrm{SiC}$ has not been studied intensively yet. Krasotkina first reported ${ }^{21}$ that hot $\mathrm{HF} / \mathrm{HNO}_{3}$ mixture is able to etch the cubic form of $\mathrm{SiC}$,

$$
\begin{aligned}
\mathrm{SiC}+2 \mathrm{HNO}_{3}+6 \mathrm{HF} \rightarrow & \mathrm{H}_{2} \mathrm{SiF}_{6}+2 \mathrm{HNO}_{2} \\
& +\mathrm{CO}_{2}+2 \mathrm{H}_{2} .
\end{aligned}
$$

In this reaction two gas phase products are formed, namely, carbon dioxide and hydrogen. Both components have very low solubility in acids even at high temperature $^{22,23}$ and ambient pressure. Thus, they can induce bubble formation on the $\mathrm{SiC}$ surface. These bubbles mask the $\mathrm{SiC}$ surface and most likely staunch the formed pores. The higher pressure increases the solubility of gases ${ }^{24}$ and the diffusion of the bubbles away from the surface takes place more easily. The generation of relatively small pores during etching is possible in this way, which may lead to a narrower size distribution. Deeper understanding of wet etching of $\mathrm{SiC}$ with $\mathrm{HF} / \mathrm{HNO}_{3}$ may come from the studies of isotropic etching of silicon using the same mixture. The $\mathrm{HF} / \mathrm{HNO}_{3}$ etching system is one of the most widely used isotropic etchant for silicon, but reduction of nitrogen during the reaction shows very complex chemistry. Most of the authors agree that the active agent of silicon etching is not $\mathrm{NO}_{3}{ }^{-}$, but an intermediate nitrogen species in the oxidation state $+3 .{ }^{25-28}$ We suppose that the active agent is similar in the etching of $\mathrm{SiC}$ and the accelerated etching could be due to the higher intermediate stability in the closed system. In an open system, the gas phase reaction product of nitrogen reduction can escape and this can shift the equilibrium, which lowers the probability of etching. In a closed system, however, the gaseous products stay in the reaction chamber, and can react with water increasing the active intermediate concentration.

We can see evidence of these theories. In reaction (1) nitrous acid $\left(\mathrm{HNO}_{2}\right)$ is the final product that is stable only in very dilute and cold conditions. During the reactions, brown nitrogen dioxide gas is formed which is visible in an open system and also when the closed system is opened. The digestion bomb is under ambient pressure when cooled back to room temperature, thus most of the produced nitrogen oxide gases can react with water or can dissolve in it. The strong yellow color of the acid from the digestion bomb instead of light yellow or colorless acid in an open system is a well-known sign for a higher concentration of dissolved nitrogen dioxide.

All in all, the reaction chamber helps the active agent to be present at higher concentration in the solvent, which leads to smaller sized pores, thus smaller sized nanoparticles. In addition, the output of nanosized $\mathrm{SiC}$ particles is also increased. Further optimization of parameters is in progress. Next, we turn to the characterization of our samples that are highly important in future bioapplications.

\section{A. Surface IR analysis}

The presence of surface carboxylic groups is crucial for the biofunctionalization of the SiC QDs and the study of the surface of these QDs is of great importance. Attenuated total reflectance (ATR) infrared spectroscopy can be used for the characterization of the surface structure of SiC QDs. ATR measurements were carried out after solvent evaporation from the ATR crystal surface. Figure 2(a) presents the IR spectra of SiC QDs synthesized in an open reaction chamber at ambient pressure. The IR spectra of SiC QDs dried from water and $\mathrm{EtOH}$ are shown together with the corresponding IR spectra of the pure solvents. In this way it is possible to distinguish between surface IR bands and solvent IR bands. These results were discussed in detail in our previous article ${ }^{20}$ where the average size of the SiC QDs was larger and also the presence of QDs larger than $10 \mathrm{~nm}$ was shown by HRTEM. In comparison, in the present study smaller SiC QDs were synthesized and studied and no QDs above $10 \mathrm{~nm}$ were detected. Figure 2(b) shows the dried $\mathrm{SiC}$ QDs from water and ethanol (EtOH) in comparison with the spectrum of SiC MCs. Here we discuss just the lowfrequency region below $1800 \mathrm{~cm}^{-1}$, because of the presence of characteristic $\mathrm{C}=\mathrm{O}$ related bands in this region. The highfrequency region above $2700 \mathrm{~cm}^{-1}$ usually contains the $\mathrm{C}-\mathrm{H}$ and possible $\mathrm{O}-\mathrm{H}$ or $\mathrm{N}-\mathrm{H}$ stretching vibrations and was discussed in our previous work. ${ }^{20}$ The $\mathrm{Si}-\mathrm{C}$ band at $\sim 800 \mathrm{~cm}^{-1}$ is present for each sample. The IR spectrum of $\mathrm{SiC}$ MCs contains only the $\mathrm{Si}-\mathrm{C}$ band [Fig. 2(b), black curve]. For SiC QDs, due to the higher surface-to-volume ratio, the surface structure is different from $\mathrm{SiC} \mathrm{MCs}$ and is clearly revealed in Fig. 2(b). Among the surface-related bands we also expect the presence of the IR bands of the remnant pure solvents; however, clear evidence of the $\mathrm{SiC}$ QDs surface bands are present in these spectra as already described earlier. ${ }^{20}$ The IR spectrum of pure water contains two strong bands at $\sim 3400$ and $\sim 1640 \mathrm{~cm}^{-1}$. The band 
at $1593 \mathrm{~cm}^{-1}$ of the $\mathrm{SiC}$ QDs dissolved in water [Fig. 2(b), blue curve] can be assigned to the remnant $\mathrm{O}-\mathrm{H}$ bending vibrations of the pure solvent, but this is the region where surface $\mathrm{C}=\mathrm{O}$ stretching vibrations are also expected. The presence of different carbonyl or carboxylate groups on the surface of $\mathrm{SiC}$ QDs becomes evident in the case where $\mathrm{EtOH}$ is used as solvent [Fig. 2(b), red curve]. Pure EtOH does not show any characteristic band in the region 1500$1800 \mathrm{~cm}^{-1}$. The presence of the band at $1732 \mathrm{~cm}^{-1}$ together with the band at $1260 \mathrm{~cm}^{-1}$ indicates the symmetric and asymmetric COO-stretching vibrations and is in good agreement with theoretical calculations. ${ }^{29}$ In the region between 1000 and $1200 \mathrm{~cm}^{-1}$, most likely the Si-O-Si and $\mathrm{C}-\mathrm{O}-\mathrm{C}$ surface vibrations can be seen. $\mathrm{Si}-\mathrm{H}$ vibrations could not be detected; thus they are not representative of our fabricated $\mathrm{SiC}$ QDs. Theory suggests that $\mathrm{Si}-\mathrm{O}-\mathrm{Si}$ bonds are

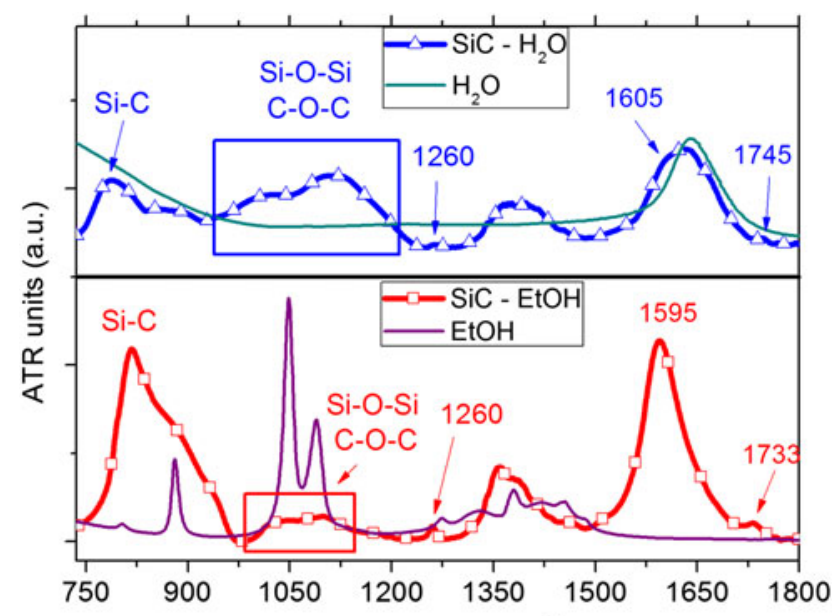

(a)

Wavenumber $\left(\mathrm{cm}^{-1}\right)$

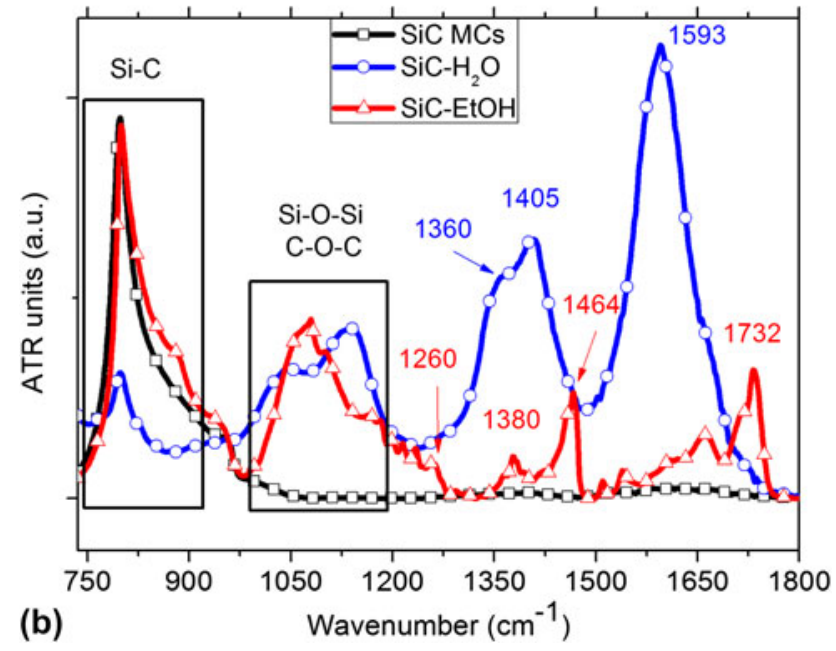

FIG. 2. (a) IR spectra of SiC QDs in water and $\mathrm{EtOH}$ in comparison with pure water and $\mathrm{EtOH}$, respectively. SiC QDs were synthesized in an open reaction chamber at ambient pressure. (b) IR spectra of SiC QDs synthesized in a closed reaction chamber at high pressure. The IR spectrum of the starting SiC MCs is shown for comparison. Each spectrum was baseline corrected and scaled to enhance visibility. more stable than $\mathrm{Si}-\mathrm{H}$ bonds on $\mathrm{SiC}$ QDs which is in line with our finding. ${ }^{30}$ Further surface-related bands are present at $\sim 1350$ and at $\sim 1400 \mathrm{~cm}^{-1}$ for each colloid. These bands are assigned to the surface related $\mathrm{CH}$ and $-\mathrm{OH}$ bending vibrations. The clear differences in the IR spectra of the two studies are related to the more evident presence of carboxylate groups on the surface of SiC QDs when dissolved in $\mathrm{EtOH}$ and QD-related - $\mathrm{OH}$ groups in water.

\section{B. Photoluminescence study}

PL spectra of the SiC QDs were taken in water and ethanol solution in the 300-400 $\mathrm{nm}$ excitation range. The highest band intensity appears at an excitation of 340-370 nm with the corresponding emission in the range of $430-450 \mathrm{~nm}$ [Fig. 3(a) upper panel-for the aqueous colloidal solution].
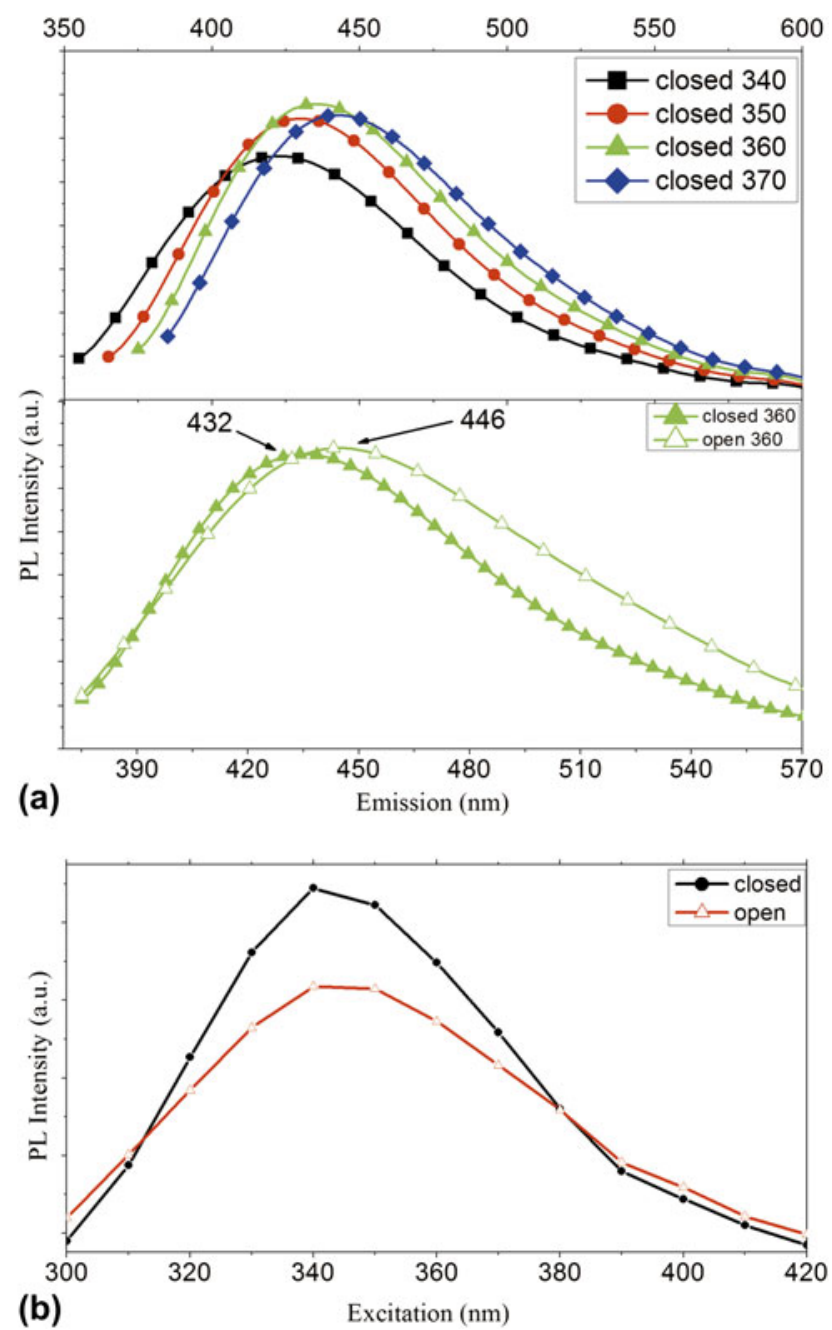

FIG. 3. (a) PL spectra of aqueous $\mathrm{SiC}$ QDs prepared in closed high pressure reaction chamber (upper panel) at excitation $340,-350,-360$, and $370 \mathrm{~nm}$. PL spectra of the samples prepared by the two methods at excitation with $360 \mathrm{~nm}$. The spectra were scaled for a better visualization (lower panel). (b) Comparison of the PL intensity at different excitation wavelengths for the two methods. 


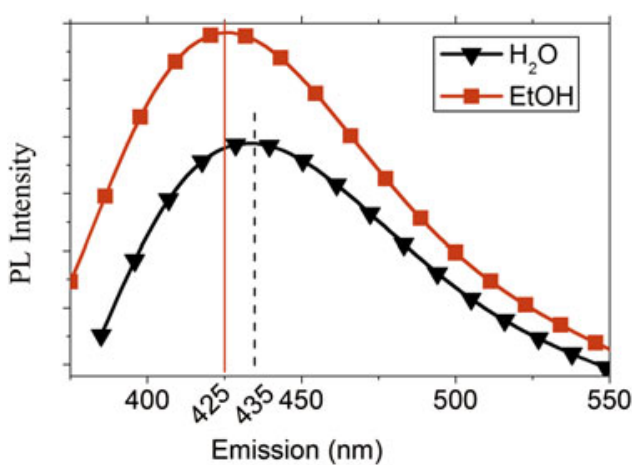

FIG 4. PL spectra of SiC QDs in aqueous and ethanol solution. Straight (dashed) vertical line shows the maximum intensity of $\mathrm{H}_{2} \mathrm{O}(\mathrm{EtOH})$ solution.

In comparison with our previous study where an open system was used for synthesizing the $\mathrm{SiC} \mathrm{QDs},{ }^{20}$ the PL spectrum of $\mathrm{SiC}$ QDs synthesized in a closed system shows two major differences. There is a slight blue shift in the emission maximum and the spectrum is generally less broad for $\mathrm{SiC}$ QDs produced in the closed reaction chamber than those in the open container [Fig. 3(a) lower panel]. Both differences confirm narrower size distribution in accordance with the HRTEM micrographs and show a nearly monodisperse distribution range (1-8 $\mathrm{nm}$ for the closed system instead of $1-15 \mathrm{~nm}$ for the open system) and a lower average size ( $3 \mathrm{~nm}$ instead of $6 \mathrm{~nm}$ ). To compare the absolute intensities of the PL emission intensities at different excitations for the two systems a new set of experiments was performed where all experimental parameters were kept the same. The result is shown in Fig. 3(b). The PL intensity is about 30\% stronger for the closed reaction chamber prepared $\mathrm{SiC}$ QDs, which is in line with our findings related to the enhanced yield production.

Comparing the PL spectra recorded in water and $\mathrm{EtOH}$ solution, a red shift with increasing solvent polarity can be observed (Fig. 4). The concentration and size distribution of the samples was the same, as well as the setup parameters. We mostly attributed this behavior to the change in the dielectric constant of different solvents. ${ }^{20}$

\section{SUMMARY}

We developed an inexpensive technology for preparing ultrasmall $\mathrm{SiC}$ nanoparticles for in vivo biomarker applications. We found that it is crucial to increase the concentration of the active agent in the solvent for increasing the output of nanoparticles and reduce their size. We were able to achieve this by applying a closed reaction chamber. Photoluminescence studies showed efficient emission in the blue-green visible range upon violet excitation. Surface-sensitive infrared spectroscopy revealed the presence of carboxyl groups at the surface of our $\mathrm{SiC}$ nanoparticles that could throw the door open for biofunctionalization. Further work relating to the development of our technology and biological tests are in progress.

\section{ACKNOWLEDGMENTS}

The Hungarian National Research Fund (OTKA Grant No. 101819) and Bolyai János Research Scholarship of HAS for Zs.C. are acknowledged.

\section{REFERENCES}

1. F. Brandizzi, M. Fricker, and C. Hawes: A greener world: The revolution in plant bioimaging. Nat. Rev. Mol. Cell Biol. 3, 520-530 (2002).

2. I.L. Medintz, H.T. Uyeda, E.R. Goldman, and H. Mattoussi: Quantum dot bioconjugates for imaging, labeling and sensing. Nat. Mater. 4, 435-446 (2005).

3. R. Hardman: A toxicologic review of quantum dots: Toxicity depends on physicochemical and environmental factors. Environ. Health Perspect. 114, 165-172 (2006).

4. C.T. Raya, D.H. Maldonado, J.R. Rico, C.G. Gañan, A.R. de Arellano-Lopez, and J.M. Fernandez: Fabrication, chemical etching, and compressive strength of porous biomimetic $\mathrm{SiC}$ for medical implants. J. Mat. Res. 23, 3247-3254 (2008).

5. C. Coletti, M.J. Jaroszeski, A. Pallaoro, A.M. Hoff, S. Iannotta, and S.E. Saddow: Biocompatibility and wettability of crystalline $\mathrm{SiC}$ and Si surfaces. In IEEE EMBS Proceedings 29th Annual International Conference. 5849-5852 (EMBS, Lyon, France,2007).

6. C.L. Frewin, M. Jaroszeski, E. Weeber, K.E. Muffly, A. Kumar, M. Peters, A. Oliveros, and S.E. Saddow: Atomic force microscopy analysis of central nervous system cell morphology on silicon carbide and diamond substrates. J. Mol. Recognit. 22, 380-388 (2009).

7. S. Wang, C. Zhang, Z. Wang, and X. Zu: Quantum confinement effect in silicon carbide nanostructures: A first principles study. Optoelectron. Rel. Mater. 4(6), 771-773 (2006).

8. M. Vörös, P. Deák, T. Frauenheim, and A. Gali: The absorption spectrum of hydrogenated silicon carbide nanocrystals from $a b$ initio calculations. Appl. Phys. Lett. 96, 051909 (2010).

9. D.H. Feng, Z.Z. Xu, T.Q. Jia, X.X. Li, and S.Q. Gong: Quantum size effects on exciton states in indirect-gap quantum dots. Phys. Rev. B 68, 035334 (2003).

10. X. Wu, J. Fan, T. Qiu, X. Yang, G. Siu, and P.K. Chu: Experimental evidence for the quantum confinement effect in $3 \mathrm{C}-\mathrm{SiC}$ nanocrystallites. Phys. Rev. Lett. 94, 6-9 (2005).

11. J. Botsoa, J.M. Bluet, V. Lysenko, O. Marty, D. Barbier, and G. Guillot: Photoluminescence of $6 \mathrm{H}-\mathrm{SiC}$ nanostructures fabricated by electrochemical etching. J. Appl. Phys. 102, 083526 (2007).

12. J. Botsoa, J. Bluet, V. Lysenko, L. Sfaxi, Y. Zakharko, O. Marty, and G. Guillot: Luminescence mechanisms in $6 \mathrm{H}-\mathrm{SiC}$ nanocrystals. Phys. Rev. B 80, 1-6 (2009).

13. J.Y. Fan, X.L. Wu, H.X. Li, H.W. Liu, G.G. Siu, and P.K. Chu: Luminescence from colloidal 3C-SiC nanocrystals in different solvents. Appl. Phys. Lett. 88, 041909 (2006).

14. J. Botsoa, V. Lysenko, A. Géloën, O. Marty, J.M. Bluet, and G. Guillot: Application of 3C-SiC quantum dots for living cell imaging. Appl. Phys. Lett. 92, 173902 (2008).

15. J. Fan, X. Wu, P. Zhao, and P.K. Chu: Stability of luminescent 3C-SiC nanocrystallites in aqueous solution. Phys. Lett. A 360, 336-338 (2006).

16. Z. Makkai, B. Pécz, I. Bársony, G. Vida, A. Pongrácz, K.V. Josepovits, and P. Deák: Isolated $\mathrm{SiC}$ nanocrystals in $\mathrm{SiO}_{2}$. Appl. Phys. Lett. 86(25), 253109 (2005).

17. J.G.C. Veinot, E.J. Henderson, and C.M. Hessel: Sol-gel derived precursors to group 14 semiconductor nanocrystals - Convenient materials for enabling nanocrystal- based applications. Mat. Sci. Eng. 6, 012017 (2009). 
18. J. Fan, X. Wu, and P.K. Chu: Low-dimensional SiC nanostructures: Fabrication, luminescence, and electrical properties. Prog. Mater Sci. 51(8), 983-1031 (2006).

19. J. Zhu, Z. Liu, X.L. Wu, L.L. Xu, W.C. Zhang, and P.K. Chu: Luminescent small-diameter $3 \mathrm{C}-\mathrm{SiC}$ nanocrystals fabricated via a simple chemical etching method. Nanotechnology 18, 365603 (2007).

20. D. Beke, Zs. Szekrényes, I. Balogh, M. Veres, É. Fazakas, L.K. Varga, K. Kamarás, Zs. Czigány, and A. Gali: Characterization of luminescent silicon carbide nanocrystals prepared by reactive bonding and subsequent wet chemical etching. Appl. Phys. Lett. 99, 213108 (2011)

21. N.I. Krasotkina, V.C. Yakovleva, N.I. Voronin, S.P. ShmittFogelevich: Stability of Silicon Carbide to Hydrofluoric, Nitric, and Sulfuric Acids, in Ogneupory (in russian) 11, 49 (1968).

22. J.D. Cox and A.J. Head: Solubility of carbon dioxide in hydrofluoric acid solutions. Trans. Faraday Soc. 58, 18391845 (1962).

23. P. Ruetschi and R.F. Amlie: Solubility of hydrogen in potassium hydroxide and sulfuric acid. J. Phys. Chem. 70(3), 718-723 (1966).
24. R. Wiebe and V.L. Gaddy: The solubility of carbon dioxide in water at various temperatures from 12 to $40^{\circ}$ and at pressures to 500 atmospheres. J. Am. Chem. Soc. 62(4), 815-817 (1940).

25. H. Robbins and B. Schwartz: Chemical etching of silicon. J. Electrochem. Soc. 106(6), 505-508 (1959).

26. M. Steinert, J. Acker, A. Henßge, and K. Wetzig: Etching of silicon in HF/HNO3 mixtures. J. Electrochem. Soc. 152(12), C843-C850 (2005).

27. M. Steinert, J. Acker, and K. Wetzig: New aspects on the reduction of nitric acid during wet chemical etching of silicon in concentrated $\mathrm{HF} / \mathrm{HNO}_{3}$ mixtures. J. Phys. Chem. C 112, 14139-14144 (2008).

28. M. Steinert, J. Acker, M. Krause, S. Oswald, and K. Wetzig: Reactive species generated during wet chemical etching of silicon in $\mathrm{HF} / \mathrm{HNO} 3$ mixtures. J. Phys. Chem. B 110(23), 11377-11382 (2006).

29. M. Vörös, P. Deák, T. Frauenheim, and Á. Gali: The absorption of oxygenated silicon carbide nanoparticles. J. Chem. Phys. 133, 064705 (2010).

30. M. Vörös, P. Deák, T. Frauenheim, and Á. Gali: Influence of oxygen on the absorption of silicon carbide nanoparticles. Mater. Sci. Forum 679-680, 520 (2011). 